\title{
Single tooth edentation caused by missing of the 6-year mollar (maxillary and/or mandible) - consequences and fix prosthetic treatment solutions
}

Irina Adriana BEURAN ${ }^{1}$, Ileana IONESCU ${ }^{1}$, Oana EFTENE ${ }^{1}$, Cosmin MEDAR ${ }^{1}$, Maria Glencora COSTACHE ${ }^{1}$, Corina Marilena CRISTACHE ${ }^{1}$, Liliana BURLIBASA ${ }^{2}$, Viorel Stefan PERIEANU ${ }^{1}$, Madalina Violeta PERIEANU ${ }^{1}$, Iuliana BABIUC ${ }^{1}$, Oana Cella ANDREI ${ }^{1}$, Luminita OANCEA ${ }^{1}$, Simion Gheorghe DUMITRU ${ }^{3}$, Gabriela TANASE ${ }^{1}$

1 "Carol Davila" University of Medicine and Pharmacy, Bucharest, Romania

${ }^{2}$ Faculty of Biology, University of Bucharest, Romania

${ }^{3}$ Dental practice, Brașov, Romania

\begin{abstract}
The loss of first permanent molars, maxillary and/or mandibular, can lead in time, due to the lack of prosthetic restoration, to a series of complications, some of high gravity, which can cause not only severe difficulties in subsequent prosthetic rehabilitation, but also substantial additional costs for patients.

Purpose. Thus, in this material we tried to bring to the attention of dental practitioners the most truthful arguments, for a fixed prosthetic restoration as early and efficient as possible.

Material and method. 53 dental practitioners, from different cities of the country, took part in this study conducted between May 2017 and April 2019.

Results and discussions. The answers obtained by applying the questionnaire were analyzed statistically and exposed for a better understanding by clear graphical methods.

Conclusions. The restoration of this type of single tooth edentation produced by the absence of the 6-yearold molar, regardless of location (maxillary and / or mandibular), is considered by most dental practitioners to be relatively simple, regardless of the therapeutic option selected.
\end{abstract}

Keywords: single tooth edentation, 6-year-old molar, fix prosthetic treatment

\section{INTRODUCTION}

Single tooth edentation is an extremely complex condition, it is present at all ages, including very young age, and can alter most of the dento-maxillary functions: mastication, swallowing, phonation and physiognomy. This type of edentation, although not always taken into account by patients, unless it is localized in the frontal region and clearly affects the physiognomy, may be accompanied by more or less clinically detectable complications. But, the most obvious of these complication is dental displacement as a result of losing dental contact points of teeth limiting edentation (1-4). 
The main causes of tooth loss in edentations of any kind, including single tooth edentations and regardless of age, are dental caries and periodontal disease. Also among the important causes of the single tooth edentation, but with a much lower frequency, we can mention the traumas (mainly found in children at the frontal teeth), the fractures of non-vital and endodontic treated teeth, the tumors of the maxillary bones, occlusal overloads that lead to the weakening of the teeth support system, and also iatrogenic, the latter being caused by the choice of an incorrect treatment plan by the dental practitioner (1-4).

In most of the cases, the first permanent teeth that are extracted from the dental arch are the first molars or the 6 years molars $(1.6,2.6,3.6$, 4.6), as they are known in the literature. Both the maxillary and mandibular ones, due to the fact that, being the first permanent teeth that appear on the dental arch, are also the first teeth affected by caries and suffering the first dentistry treatments (1-3). Specifically, by losing the first molars both maxillary and mandibular, patients lose a significant part of the active masticatory area (1).

\section{GENERAL DATA}

The loss of the first molars (the 6-year molars), both maxillary and mandibular, can cause major changes in harmony of dental arches and functionality of the dento-maxillary system.

The effects of losing of first permanent molars can be seen both in the positioning of neighboring and antagonist teeth, as well as in mandibular movements, due to premature contacts and / or interferences, which can even lead to mandibular lateral deviations (1-4). Early loss of the 6-year-old molar also entails a series of migrations in the vertical plane of the antagonist teeth as well as in the sagittal plane of adjacent teeth. Consequences of 6-year-old molar loss at an early age cause changes in the eruption of neighboring and antagonist teeth, changes that affect their speed and direction of eruption (1-4).

Following the extraction of the 6-year-old molar and the dental migration of the teeth surrounding the edentation, premature contacts (interferences) occur during mandibular movements. Trying to avoid these interferences, the mandible will perform bruxism like paraphunctional movements, but for short term $(1,5)$. When interference has been removed through attrition, paraphunctional movements cease. The consequences of this type of temporary bruxism can be observed both at the level of causal teeth, meaning the teeth next to the edentation, as well as at the level of the frontal group, located on the diagonal of the edentation, especially at the level of the canine and the lateral incisor, but wear surfaces may also occur at the level of other teeth $(1,5)$. In the context of continuous overload, bruxism can become permanent, with serious repercussions on the balance of the dento-maxillary system $(1,5)$.

But what should be remembered in the case of both vertical and sagittal teeth movements due to the loss of first permanent molars, both maxillary and mandibular, is that they can be accompanied by periodontal lesions of varying severity, which complicates even more clinical situation (1-4). Interruption of the dental arch modifies the functional stress to which the supporting structures of adjacent teeth are subjected. Migrations of the teeth next to missing area will lead to the loss of contact points with the appearance of premature contact and interference that will disrupt neuromuscular activity. This anomaly will increase the destructive effect of the occlusal forces on the marginal periodontium, resulting in the occurrence of periodontal complications of different intensities, consisting in the formation of deep bone pockets and the occurrence of pathological dental mobility (1-4).

Also, following the loss of 6-year molars (1.6, $2.6,3.6,4.6)$, the eruption of wisdom teeth (1.8, $2.8,3.8,4.8)$ will be accelerated by the side where these teeth were extracted. For example, the mandibular wisdom tooth will erupt in a mesial position, preventing the eruption in a normal occlusal plane of the maxillary wisdom tooth. As a result of this, it can erupt in an abnormal position (rotated, toward oral side, horizontal), traumatizing the jugular mucosa (1-4).

Other early and / or late complications resulting from the extraction of 6-year-old molars (maxillary and / or mandibular) that may occur in patients who have neglected or refused any prosthetic rehabilitation of resulting edentulous space may be (1-4): changes in the vertical dimension of occlusion; lateral deviations of the mandible; reduced masticatory capacity on the side where the 6-year molar extraction was performed by up to $50 \%$; dysfunction of the temporomandibular joint; trauma of the jugular mucosa of edentulous space with its changes; digestive disorders (gastritis, ulcer, etc.) due to insufficient food trituration; psychic consequences that can often take dramatic forms.

\section{PURPOSE}

Generally, patients do not pay the same attention to the lateral single tooth edentations and do 
not consider a need for their prosthetic treatment, unlike frontal edentations, when the physiomic aspect is affected due to the lack of one or more teeth. Although, by the extraction of molars in general, $3 / 4$ of the active mastication area is lost, the loss of the posterior teeth does not always cause patients to rush up the start of a dental treatment.

The possible treatment variants for single tooth edentations produced by the extraction of the first permanent molars both maxillary and mandibular can be chosen depending on the clinical situation, but also taking into account the requirements of the patients. Thus, most patients want fixed, less invasive prostheses and which are the most effective in mastication but which do not require a long time to be made and which, last but not least, are accessible from the financially point of view, point that is not at all easy to accomplish (1-4).

The older the edentulous space is, the more its disto-mesial dimension decreases due to the migration of limiting teeth, the bone crest is thinner and more resorbed, and these complications further complicate the treatment plan, requiring many meetings and also higher costs (1-4).

There are several fixed prosthetic treatment options for single tooth edentations. Each treatment solution has advantages and disadvantages which, explained to patients, may influence the choice of the treatment plan. Although most patients want a fixed prosthetic restoration, whether conventional, winged bridge or supported on dental implants, it cannot always be obtained (1-4). Before discussing the treatment plan with the patient, the physician should assess the clinical and radiological status. And when we talk about a radiological exam, it must be performed and interpreted to the highest medical standards. There are a few criteria that are taken into account in choosing the prosthetic treatment (1-4): the age of the edentulous space; the location of the edentulous space; state of limiting teeth; bone supply, general condition of patients; the cost of prosthetic restoration.

In the case of the 6-year molar edentation, regardless of its location mandibular and/or maxillary, the fastest treatment is to obtain conventional prosthetic restorations. These can be accomplished in a shorter time, but require preparing of neighboring teeth, and in most cases root canal treatment (1-8).

Fixed implant-supported prosthetic restorations have the great advantage of not requiring the preparation of teeth limiting the edentulous space, but are a treatment method that requires a much longer period of time and much higher costs (1-4, 9-17).

Winged bridges are not under discussion in our case because, although it is done in a very short time and requires a minimal preparation of the neighboring teeth, they are used more as short term treatment solutions and the very strong masticatory pressure in this area usually dislodging them at rather short intervals.

But as an extremely interesting but also extremely important mention is that in the fixed prosthetic treatment of a single tooth edentation obtained by the absence of the first permanent molar, maxillary and / or mandibular, with classic or implant-supported prosthetic treatment, is often necessary the intervention of the specialist in orthodontics and dento-facial orthopedics either in order to open the space to insert a dental implant or to make a fixed prosthetic restoration in which the dental bridge body has dimensions within normal limits or for complete closure of edentulous space, especially when patients are children or adolescents (1-4). In most of the cases, single tooth edentations caused by the absence of 6-year molars are not immediately treated, which in time leads to diminishing the edentulous space due to the loss of interdental contact points, there are changes in the position of the teeth limiting edentulous space (inclination, rotation, translation) as well as vertical migrations of antagonist teeth, migrations consisting of extrusion or egression (1-4). Thus, the orthodontic treatment with the opening of the edentulous space has become a component increasingly popular and recommended by the dentists.

In conclusion, we can appreciate that the purpose of this study was to direct dentists to both the treatment plan and the consequences, some of which may become dramatic, to which patients who have suffered 6-year molar losses and who neglected or refused classical prosthetic or implant-supported prosthetic rehabilitation of the edentulous breach resulting from the loss of these permanent teeth.

\section{MATERIAL AND METHOD}

Starting not only from the notions presented in the first part of this paper, but also from our authors' experience, we have developed a very precise questionnaire, which contains 8 questions, aiming to guide the doctors on the treatment plan, as well as on the consequences to which patients who have suffered extractions of the first perma- 
nent molar regardless of their location maxillary or mandibular and who have neglected or refused classical prosthetic or implant-supported rehabilitation of the edentulous spaces.

This study was conducted over a period of approximately 2 years between May 2017 and April 2019. The questionnaire was applied to a number of 53 dental practitioners from several cities in the country (Bucharest, Brasov, Pitesti, Constanta, Tulcea, Ploiesti, Alexandria, Resita, Rm. Valcea) who are active in the private sector, aged between 41 and 68 , with a rather extensive practical experience. The gender distribution of the subjects involved in the study was as follows: 39 of the subjects (representing 73.58\%) were female, while 14 of the subjects (representing $26.42 \%$ ) were male (Fig. 1).

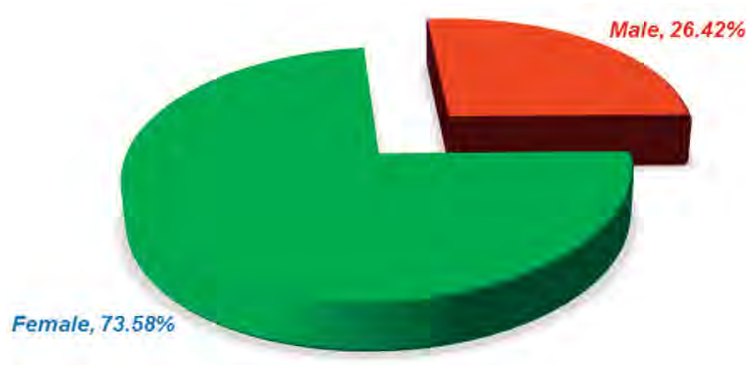

FIGURE 1. Gender distribution of study group

The questionnaire applied to the 53 subjects was the following:

\section{Questionnaire}

1. In patients who have suffered 6-year-old molar loss, may be observed over longer or shorter time periods, the following aspects: $\boldsymbol{a}$. The eruption of the wisdom teeth will be accelerated on the side where these teeth have been extracted; $\boldsymbol{b}$. There are changes in the vertical dimension of the occlusion; c. The masticatory capacity is substantially reduced; $\boldsymbol{d}$. Traumas and alterations of the jugular mucosa beside the edentulous space may occur; e. Are there significant esthetic changes in the edentulous space.

\section{Correct answers: $a, b, c$.}

2. What negative consequences for patients do you think the loss of 6-year old molars, regardless of localization (maxillary and/or mandibular) may have? a. Migrations in the sagittal plane of the adjacent teeth and vertical of the antagonist teeth; $\boldsymbol{b}$. Temporary bruxism; c. Periodontal complications and temporomandibular joint dysfunction; d. Endocrine diseases such as diabetes mellitus; e. Digestive disorders and often neuro-psychiatric disorders.

Correct answers: $a, b, c, e$.
3. Treatment alternatives for single tooth edentations produced by the extraction of 6-year-old molars localized maxillary and/or mandibular are? a. Conventional prosthetic restoration (classic); $\boldsymbol{b}$. Partial skeletal dentures; c. Implant-supported fixed prosthetic restorations; $\boldsymbol{d}$. Partial acrylic dentures.

Correct answers: $\boldsymbol{a}, \boldsymbol{c}$.

4. The advantages of conventional (classic) prosthetic restorations that make them more acceptable by patients are as follows? a. Presents the advantage of preparing teeth limiting edentulous space; $\boldsymbol{b}$. Increased masticatory efficiency; $\boldsymbol{c}$. Affordable price; $\boldsymbol{d}$. It does not require very long time to be made.

Correct answers: $b, c, d$.

5. Among the advantages of implant-supported restorations, must be mentioned: $\boldsymbol{a}$. It does not require preparing teeth limiting edentulous space; b. Requires a much longer manufacturing time compared to conventional (fixed) prosthetic restoration; c. Costs are much higher compared to conventional (fixed) prosthetic restoration.

\section{Correct answers: $\boldsymbol{a}$.}

6. Winged bridge restorations: $\boldsymbol{a}$. Are treatment solutions with a high rate of success; $\boldsymbol{b}$. Requires root canal treatment and preparation of teeth limiting edentulous space; c. Are temporary solutions, which are not recommended for long term use.

\section{Correct answers: $c$.}

7. The criteria that a dentist should take into account when choosing a fix prosthetic treatment for restoring the 6-year molar edentation are as follows? $\boldsymbol{a}$. The age of the edentulous space; $\boldsymbol{b}$. Location of the edentulous space; c. Condition of neighboring teeth and bone supply; $\boldsymbol{d}$. The general condition of the patient; $\boldsymbol{e}$. Costs of prosthetic restoration.

\section{Correct answers: $a, b, c, d, e$.}

8. In the fixed treatment of a single tooth edentation obtained by the absence of the 6-year old maxillary and/or mandibular molar (classical or implant-supported prosthetic treatment), the dentist often asks for the intervention of the specialist in orthodontics and dentofacial orthopedics to? $\boldsymbol{a}$. Open the space to insert a dental implant or to perform a fixed prosthetic restoration in which the bridge body has normal dimensions; b. Complete closure of edentulous space, especially when patients are children or adolescents; $c$. To solve the open bite.

Correct answers: $a, b$. 


\section{RESULTS AND DISCUSSIONS}

The results obtained by applying the questionnaire to the 53 respondents were centralized and analyzed.

For the first question related to the short and long term aspects observed in patients who suffered 6-year molar loss, 41 of the subjects (representing $77.36 \%$ ) responded correctly (variants $a, b$ and $c$ respectively: the accelerated eruption of the wisdom teeth, changes in the vertical size of occlusion, and substantial reduction in masticatory capacity). The remaining 12 subjects (representing $22.64 \%$ ), in addition to the correct variants, also chose the variants $d$ or e (Fig. 2).

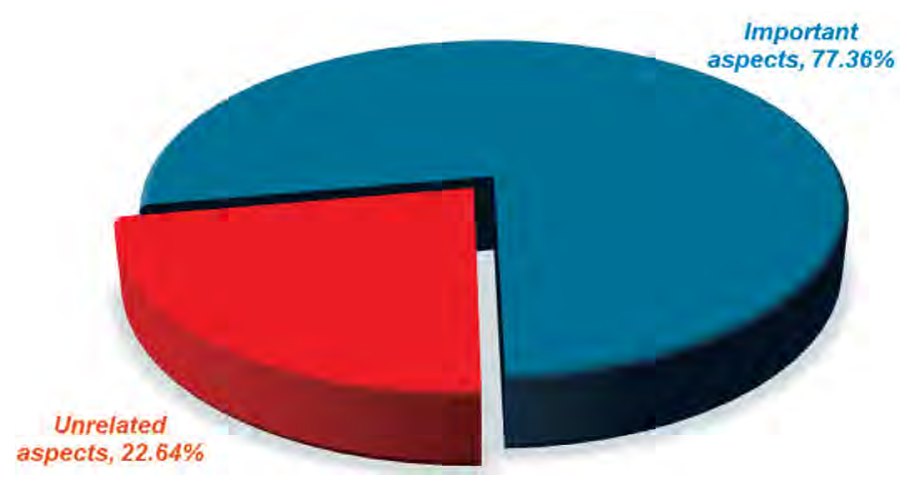

FIGURE 2. Short and long term aspects in patients with 6-year molar loss

Regarding the negative consequences that the loss of the 6-year-old molar may have for patients, the majority of respondents (43 representing $81.13 \%)$ responded correctly, namely: sagittal and vertical teeth migrations, temporary bruxism, periodontal complications, and temporomandibular joint dysfunction, respectively digestive and neu- ro-psychic disorders. The remaining 10 respondents (representing 18.87\%), provided other combinations of answers being considered totally wrong (Fig. 3).

For the third question related to fixed treatment alternatives for edentations caused by the absence of 6-year-old molars, we also find that most practitioners responded correctly. Thus, 44 of them (representing $83.02 \%$ ) responded to classical prosthetic restoration or implant-supported. The rest of the practitioners, 9 - representing $16.98 \%$, responded wrongly by choosing all the variants presented (Fig. 4).

The advantages of classical prosthetic restorations were correctly appreciated by most of the specialists included in the study, so 50 of them (representing 94.34\%) gave variants masticatory efficiency, financial accessibility and relatively short time production. Only 3 respondents representing 5.66\% responded wrongly (Fig. 5).

All participants in the study responded correctly about the benefits of implant-supported restorations. It was noted that among the participants in the study only half ( 27 - representing $50.94 \%$ ) know the concept and applications of winged bridge. The remaining 26 participants (representing $49.06 \%$ ) responded incorrectly by choosing all variants of response (Fig. 6).

Regarding the criteria underlying the choice of fixed prosthetic treatment, most practitioners (46 - representing $86.79 \%$ ) responded correctly, namely: the age and location of the edentulous space, the state of the neighboring teeth, the general condition of the patient and the costs involved. The rest of the practitioners ( 7 - representing $13.21 \%$ ) responded wrongly by incomplete selection of answers (Fig. 7).

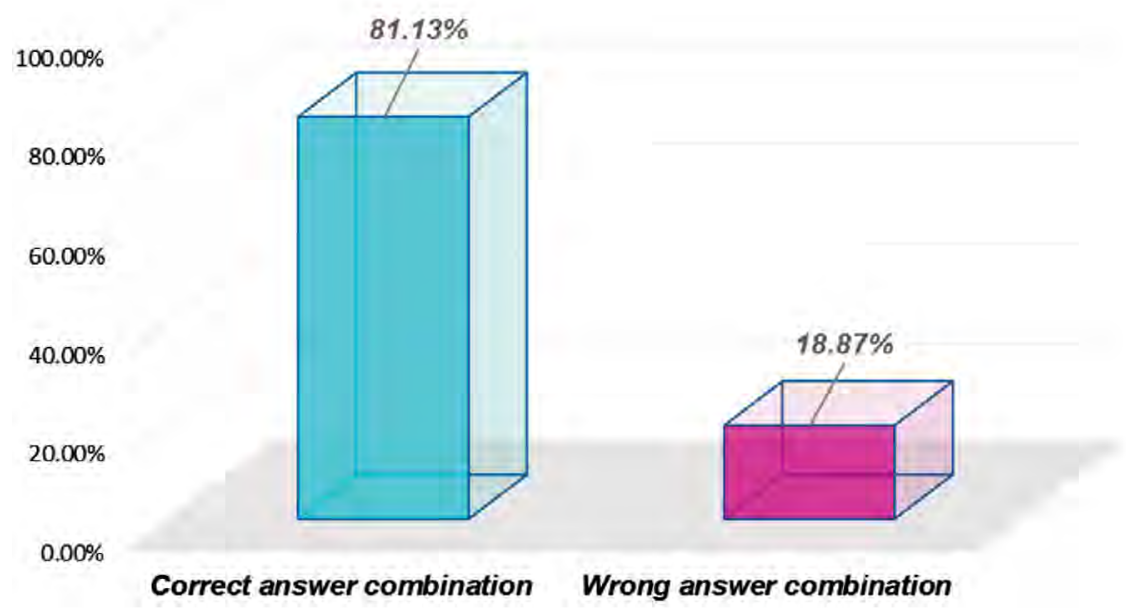

FIGURE 3. Negative consequences of 6-year-old molar loss 


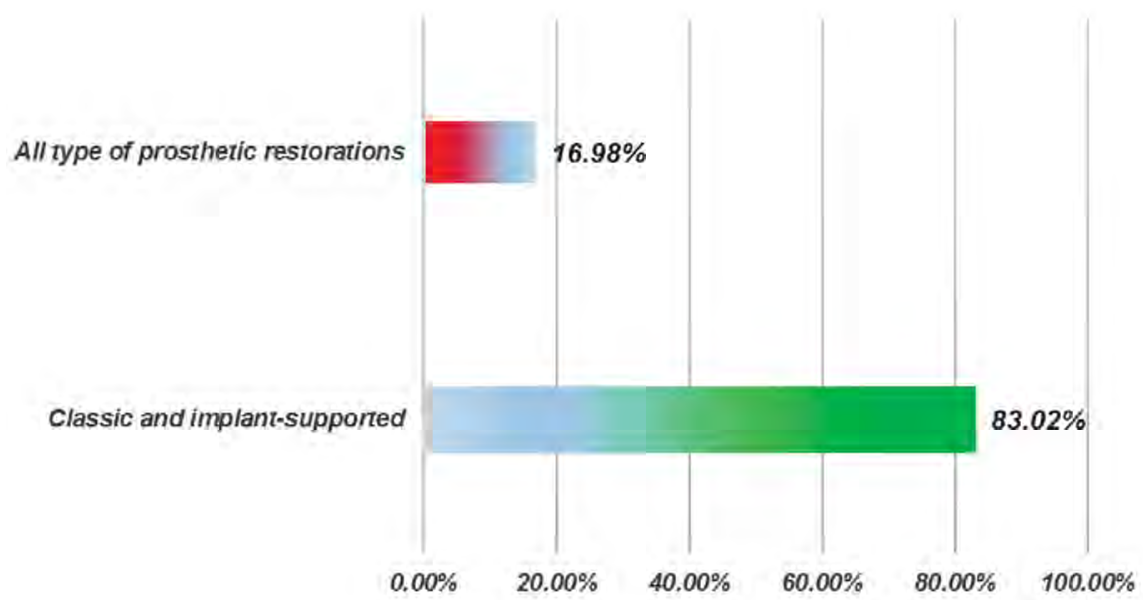

FIGURE 4. Fixed prosthetic treatment alternatives

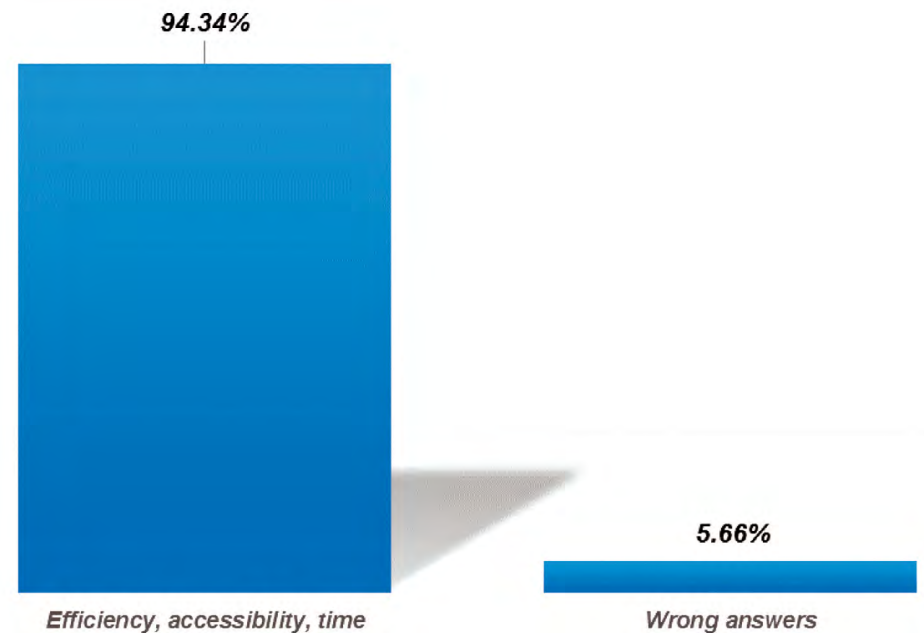

FIGURE 5. Advantages of classical prosthetic restorations

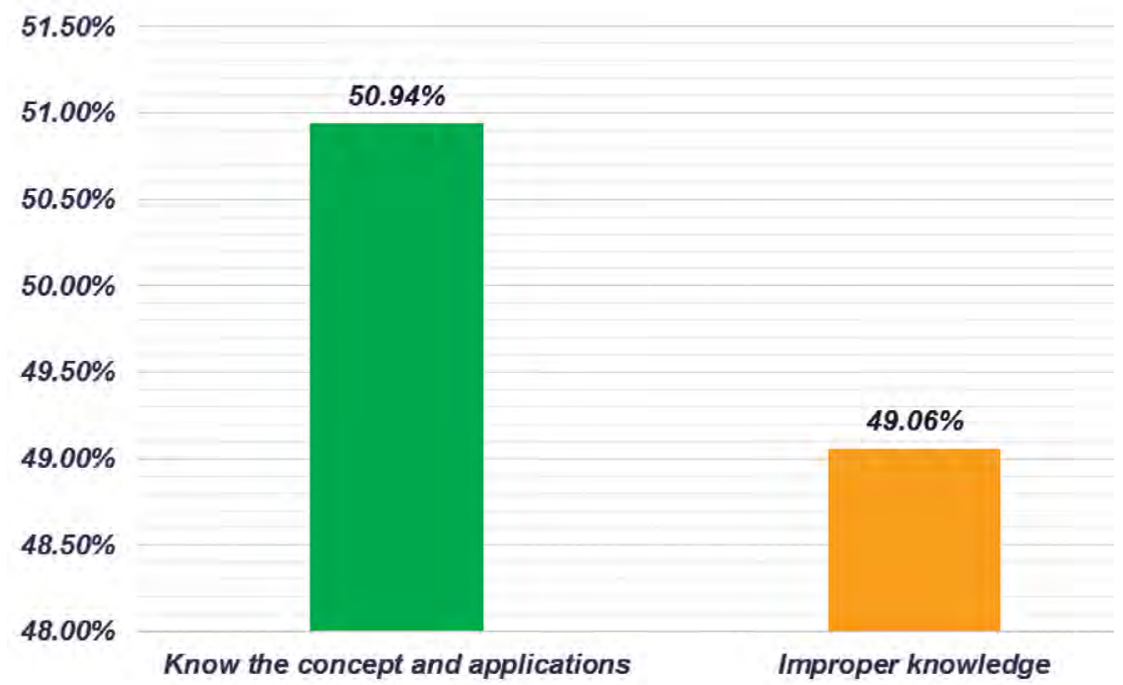

FIGURE 6. Winged bridges restorations 


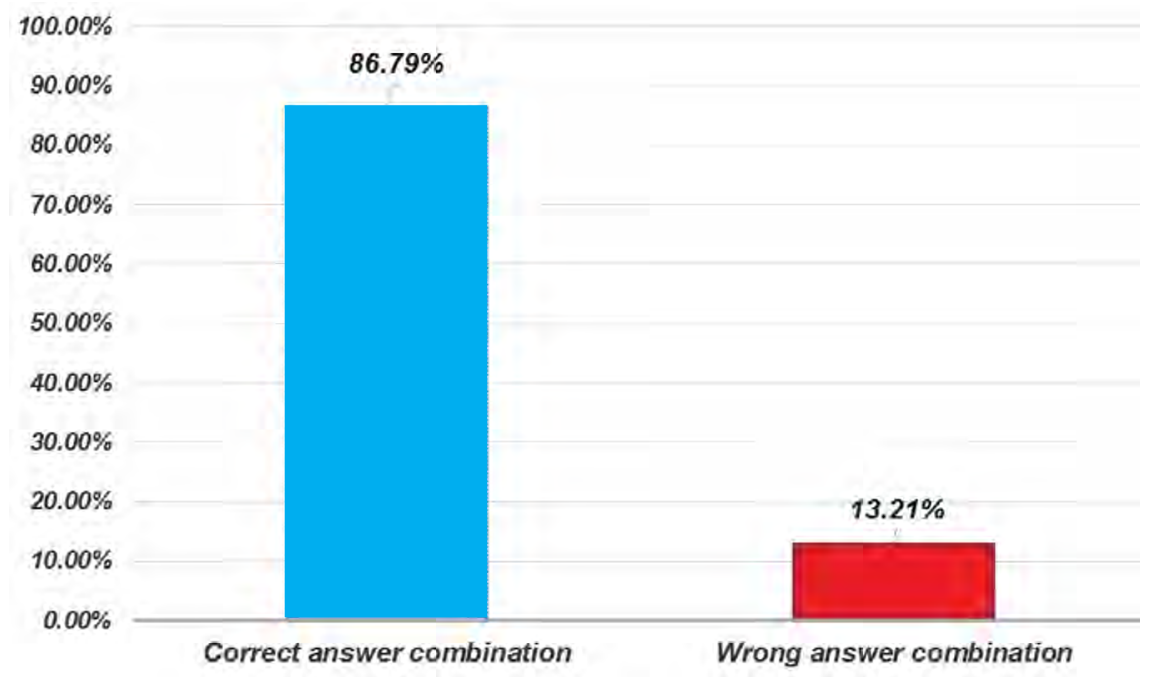

FIGURE 7. Criteria underlying the choice of fixed prosthetic treatment

For the last question of the study related to the intervention of the orthodontist, correct answers were obtained from all participants in the study.

\section{CONCLUSIONS}

The restoration of this type of single tooth edentation, produced by the absence of the sixyear-old molar, regardless of its localization (maxillary and / or mandibular), is considered by most of dental practitioners to be relatively simple regardless of the selected therapeutic option. Unfortunately, after evaluating the answers to the first two questions, we found that just over $3 / 4$ of the dentists included in the study (over 75\%) have real and thorough knowledge of the adverse effects produced by the absence of the first permanent molar, maxillary and/or mandibular (1.6, 2.6, 3.6, 4.6).

Following evaluation of fixed treatment alternatives, we found that over $15 \%$ of the subjects involved in the study failed to distinguish between fixed and mobile prosthetic restoration, which normally denotes either non-reading text or an insufficient knowledge of basic dental prosthetics. We, the authors, believe it's the inadvertently read of the text.

Regarding the advantages of conventional fixed and implant-supported prosthetic restorations, we could conclude that most of the subjects included in this study (between 95\% and 100\%) have firm knowledge of the advantages and disadvantages of using both fixed conventional as well as implant-supported prosthetic restorations. However, as a mention, there is a $5.66 \%$, which is considered insignificant from our point of view, which considers that preparing the teeth limiting the edentulous breach is a non-traumatic maneuver for the patients. We, the authors, believe that these dental practitioners haven't paid attention when reading the question.

Very surprising were the answers related to winged bridges: just over $50 \%$ (or $50.66 \%$ ) of the dentists involved in the study offered the correct answer, which indicates that dentists in the private network either do not use as temporary prosthetic solutions winged bridges, adhesive techniques in lateral areas, or have insufficient knowledge of these terms. It should be mentioned, however, that the adhesive techniques at this time represent the most suitable therapeutic solutions for patients when they are likely to be implemented but it seems that not the same can be said by dental practitioners, which many consider to be unsafe prosthetic restorations (can fracture or decimate easily in the event of a traumatic occlusion), and especially unhygienic (if proper hygiene is not ensured, there is a risk of secondary caries to appear).

When discussing the intervention of the orthodontist in the rehabilitation of a single tooth edentation obtained by the absence of the 6-year maxillary and/or mandibular molar, the conclusions can be varied, especially since the question is addressed in general terms:

an alternative would be the complete closure of the edentulous space by orthodontic treatment, but it can be applied only at very young age;

the opening of edentulous space by orthodontic treatment to insert a dental implant or to make a fixed prosthetic restoration in which the bridge body has normal dimensions is a solution that dental specialists are appealing more and more often in the last period. Although this therapeutic 
solution can be applied to all patients regardless of age, it is often denied, orthodontic treatment being a complex, long-lasting treatment (at least several months) and quite expensive. Therefore, patients are reluctant when discussing the supplementing the conventional prosthetic or implant prosthetic treatment (both fixed) with a therapeutic solution from the orthodontic area.

At this point in Romania there is a lot of discussion about a population that does not have the necessary material resources for regular dental checkups, but it is presented in the dentistry offices only when more serious problems arise. Thus, in most urban areas there are the most dental practices, the competition between them is quite serious, but unfortunately the patients are quite few. That is why many dental practitioners approach with much courage and, with quite a lot of unconsciousness, almost any kind of therapeutic solution without having the necessary knowledge and experience. Therefore, there are also plenty of mistakes, some serious, which led in recent years to many lawsuits of patients, the reason being medical malpractice.

\section{Acknowledgement}

In this article, all authors have equal contribution with the first author.

\section{Conflict of interest: none declared} Financial support: none declared

\section{REFERENCES}

1. Dina MN, Andrei OC, lonescu I et al. Aspecte teoretice și practice în tratamentul clasic și modern al edentației unidentare. În: Tănase G, Dina MN, Dumitru SG et al. Probleme în medicină și biologie, Vol. IX, București: Editura Ars Docendi, 2019: 165-312.

2. Rosenstiel SF, Land MF, Fujimoto J. Contemporary fixed prosthodontics. 4th ed. St. Louis: Mosby Elsvier, 2006.

3. Anusavice KJ. Dental materials. Philips' Science 11th ed. St. Louis: Saunders Elsvier, 2003.

4. Miyasaki-Ching CM. Elemente clinice de stomatologie. București: Ed. All, 2001.

5. Davies SJ, Gray RJM, Mackie IC. Good occlusal practice in children's dentistry. Brit Dent J. 2001; Vol. 191, No. 12: 655-659.

6. Burlibasa L., Zarnescu O. In vivo effects of Trichostatin A - a histone deacetylase inhibitor on chromatin remodeling during Triturus cristatus spermatogenesis. Anim Reprod Sci. 2013; 142 (1-2): 89-99.

7. Gavrila L, Mircea L. Chromatin and chromosomal fine structure in spermatogenesis of some species of amphibians. Zygote. 2001; 9(3): 183-192.
8. Cristache CM, Burlibasa M, Cristache $G$ et al. Zirconia and its biomedical applications. Metalurgia International. 2011; Vol. XVI, No. 7: 18-23.

9. Burlibasa M, Muntianu LAS, Tanase $\mathrm{G}$ et al. Study on microbial contamination of biomaterials in medical practice. Metalurgia International. 2010; Vol. XV, Spec. Iss. 2: 163-166.

10. Burlibasa M, Cristache CM, Georgescu SR et al. Toxicity of titan and nikel-cobaltchromium alloys. Metalurgia International. 2009; 14: 20-22.

11. Burlibasa L, Gavrila L. Chapter 6 Developmental epigenetics: roles in embryonic development. In: Niculescu MD, Haggarty P. Nutrition in Epigenetics. Willey-Blackwell Publishing 2011: 105-126.

12. Cristache $\mathrm{CM}$, lonescu $\mathrm{C}$, Cristache $\mathrm{G}$ et al. A 5-year prospective randomised clinical trial on the efficiency of two different attachement systems as retention for implant-supported mandibular overdenture. Radiographic assesment, cost analysis and final evaluation of treatment, success. Metalurgia International. 2009; Vol. XIV, Spec. Iss. No. 16: 27-34.
13. Cristache $\mathrm{CM}$, lonescu $\mathrm{C}$, Burlibasa $\mathrm{M}$ et al. Rettentive anchors versus magnets as attachment systems for mandibular overdenture. A 5 year prospective randomised clinical study. Metalurgia International. 2009; Vol. XIV, Spec. Iss. No. 16: 59-64.

14. Tanase G, Burlibasa M, Muntianu L et al. Testing the antibacterial potential of biomaterials in medical practice. Metalurgia International. 2010; Vol. XV, Spec. Issue No. 2: 160-162.

15. Burlibasa M., Cernusca-Mitariu M., Burcea $\mathrm{CC}$ et al. Halogen compounds - theoretical, physiological and practical aspects regarding the decontamination, disinfection and sterilisation of instruments and biomaterials in dental medicine practice. Metalurgia International. 2013; Vol. XVIII, Spec. Issue No. 3: 54-57.

16. Burlibasa L, Domnariu C. Epigenetic landscape of human diseases. Acta Medica Transilvanica. 2018; 23(2): 33-37.

17. Bodnar DC, Burlibasa L, Varlan C et al. Mercury, biocompatibility and its impact on environment. Metalurgia International. 2009; 14: 95-100. 